\title{
EVEN AND ODD INTEGRAL PARTS OF POWERS OF A REAL NUMBER
}

\author{
ARTŪRAS DUBICKAS \\ Department of Mathematics and Informatics, Vilnius University, Naugarduko 24, \\ Vilnius LT-03225, Lithuania \\ e-mail:arturas.dubickas@maf.vu.lt
}

(Received 4 November, 2005; revised 15 January, 2006; accepted 19 January, 2006)

\begin{abstract}
We define a subset $\mathcal{Z}$ of $(1,+\infty)$ with the property that for each $\alpha \in \mathcal{Z}$ there is a nonzero real number $\xi=\xi(\alpha)$ such that the integral parts $\left[\xi \alpha^{n}\right]$ are even for all $n \in \mathbb{N}$. A result of Tijdeman implies that each number greater than or equal to 3 belongs to $\mathcal{Z}$. However, Mahler's question on whether the number $3 / 2$ belongs to $\mathcal{Z}$ or not remains open. We prove that the set $\mathcal{S}:=(1,+\infty) \backslash \mathcal{Z}$ is nonempty and find explicitly some numbers in $\mathcal{Z} \cap(5 / 4,3)$ and in $\mathcal{S} \cap(1,2)$.
\end{abstract}

2000 Mathematics Subject Classification. 11J71, 11R04, 11R06, 11R09.

1. Introduction. Suppose that $\alpha>1$ is a real number. Then either for any real number $\xi \neq 0$ the fractional parts $\left\{\xi \alpha^{n}\right\}$ are greater than or equal to $1 / 2$ for infinitely many $n \in \mathbb{N}$ or there exists a real number $\xi=\xi(\alpha) \neq 0$ such that $\left\{\xi \alpha^{n}\right\}<1 / 2$ for every $n \in \mathbb{N}$. The set of $\alpha>1$ for which the first possibility holds will be denoted by $\mathcal{S}$. Similarly, we will denote by $\mathcal{Z}$ the set of $\alpha>1$ for which the second possibility holds, so $\mathcal{S} \cap \mathcal{Z}=\emptyset$ and $\mathcal{S} \cup \mathcal{Z}=(1,+\infty)$. Equivalently, $\alpha \in \mathcal{Z}$ if and only if there is a real number $\xi=\xi(\alpha) \neq 0$ such that the integral parts $\left[\xi \alpha^{n}\right], n=1,2, \ldots$, are all even.

In 1968, Mahler [16] asked whether the number $3 / 2$ belongs to $\mathcal{S}$ or to $\mathcal{Z}$ (see also [20]). There are many reasons to believe that $3 / 2 \in \mathcal{S}$. Although for a 'random' pair $\xi, \alpha$ the fractional parts $\left\{\xi \alpha^{n}\right\}, n=1,2, \ldots$, are uniformly distributed in $[0,1)$ (see [13], [21]), the behavior of the sequence $\left\{\xi \alpha^{n}\right\}, n=1,2, \ldots$, for almost any 'specific' pair $\xi, \alpha$, where $\xi \neq 0, \alpha \notin \mathbb{N}$, is not known. See, however, [3], [4], [6]-[12], [22], [23] for some results in this direction. In this note, we shall collect several results about the sets $\mathcal{S}$ and $\mathcal{Z}$.

Trivially, $\{2,3,4, \ldots\} \subset \mathcal{Z}$, because, for any integer $g \geq 2$, by taking $\xi=2$ we see that the numbers $\left[2 g^{n}\right]=2 g^{n}, n=1,2, \ldots$, are all even. In general, one may expect that 'large' $\alpha$ belong to the set $\mathcal{Z}$ (see, for instance, Tijdeman's result [19] stated in Theorem 1(i) below) whereas 'small' $\alpha$ lie in $\mathcal{S}$. In connection with this, one can ask whether there exist $\alpha>\alpha^{\prime}>1$ such that $\alpha \in \mathcal{S}$ and $\alpha^{\prime} \in \mathcal{Z}$. If the answer to this question were negative then the sets $\mathcal{S}$ and $\mathcal{Z}$ would simply be two intervals. Unfortunately, the situation is not that simple, because such $\alpha$ and $\alpha^{\prime}$ do exist. We will show, for instance, that the set $\mathcal{Z}$ contains a number smaller than 1.26 and that the set $\mathcal{S}$ contains the golden mean $(1+\sqrt{5}) / 2=1.61803 \ldots$

In order to state our theorems, we recall first that $\alpha>1$ is called a Pisot number if it is an algebraic integer whose conjugates over $\mathbb{Q}$ different from $\alpha$ itself lie in the open unit disc. A Pisot number is called a strong Pisot number if it is not a rational integer and if its second largest (in modulus) conjugate is positive (see [2] and [6]). Also, 
$\alpha>1$ is called a Salem number if it is an algebraic integer whose conjugates over $\mathbb{Q}$ different from $\alpha$ itself lie in the closed unit disc $|z| \leq 1$ with at least one conjugate lying on the unit circle $|z|=1$. Finally, let $P_{\alpha}(x) \in \mathbb{Z}[x]$ denote the minimal polynomial of an algebraic number $\alpha$. Note that $P_{\alpha}(1) \leq-1$ for each $\alpha$ which is a Pisot number or a Salem number.

\section{Results.}

THEOREM 1. We have

(i) $[3,+\infty) \subset \mathcal{Z}$,

(ii) $3-2 / q \in \mathcal{Z}$ for any integer $q \geq 2$,

(iii) $\alpha \in \mathcal{Z}$ for any strong Pisot number $\alpha$,

(iv) $\alpha \in \mathcal{Z}$ for any Pisot or Salem number $\alpha$ whose minimal polynomial satisfies $P_{\alpha}(1) \leq-3$.

By Theorem 1(iv), the thirteenth smallest known Salem number $\alpha=1.2527759 \ldots$ whose minimal polynomial is

$$
P_{\alpha}(x)=x^{18}-x^{12}-x^{11}-x^{10}-x^{9}-x^{8}-x^{7}-x^{6}+1
$$

belongs to the set $\mathcal{Z}$, because $P_{\alpha}(1)=-5<-3$. (See Mossinhoff's page on Lehmer's problem http://www.cecm.sfu.ca/〜 mjm/Lehmer/lists/SalemList.html for a list of small Salem numbers.) Most of the results stated in Theorem 1 have been published earlier or follow easily from [19], [10], [11], [22]. Nevertheless, for the sake of completeness, we will give the proofs of $(i)-(i i i)$ below and derive (iv) from [10], [22].

The first example of a number $\alpha>1$ lying in the set $\mathcal{S}$ was given recently by the author in [8]: for any $d \geq 4$ one can take $\alpha>1$ that satisfies $\alpha^{d}-\alpha-1=0$. So the set $\mathcal{S}$ is nonempty. Note that if $\alpha \in \mathcal{S}$ then $\alpha^{1 / q} \in \mathcal{S}$ for each $q \in \mathbb{N}$, because the set of fractional parts $\left\{\xi \alpha^{n}\right\}$, where $n \in \mathbb{N}$, is a subset of the set $\left\{\xi \alpha^{n / q}\right\}, n \in \mathbb{N}$. The next theorem not only contains the example given above but also describes some new numbers in $\mathcal{S}$.

THEOREM 2. We have

(i) $2^{1 / q} \in \mathcal{S}$ for any integer $q \geq 2$,

(ii) $\alpha \in \mathcal{S}$ for any $\alpha>1$ which is a root of an irreducible polynomial $x^{d}-x^{r}-1$, where $0<r<d$,

(iii) $\alpha \in \mathcal{S}$ for any $\alpha>1$ which is a root of the polynomial $x^{d}-x^{m}-x^{r}+1$, where $0<r \leq m<d$, but is not a Pisot number.

Note that in case (iii) the polynomial $x^{d}-x^{m}-x^{r}+1$ is reducible. Hence the degree of $\alpha>1$ over $\mathbb{Q}$ is smaller than $d$. The requirement that $\alpha$ is not a Pisot number is necessary. If, for instance, $m=r=d-1$ then

$$
x^{d}-2 x^{d-1}+1=(x-1)\left(x^{d-1}-x^{d-2}-\cdots-x-1\right) .
$$

The polynomial $P_{\alpha}(x)=x^{d-1}-x^{d-2}-\cdots-x-1$ is irreducible and defines a Pisot number $\alpha>1$ for each $d \geq 3$. Since $P_{\alpha}(1) \leq-3$ for every $d \geq 5$, Theorem $1(i v)$ implies that $\alpha \in \mathcal{Z}$. However, for $d=3, \alpha=(1+\sqrt{5}) / 2$ belongs to $\mathcal{S}$ by Theorem $2(i i)$. All irreducible polynomials of the form $x^{d}-x^{r}-1$ have been described in [15].

Since $\sqrt{2} \in \mathcal{S}$ and $\sqrt{m} \in \mathcal{Z}$ for each integer $m \geq 4$, it is natural to ask the following:

Problem 3. Determine whether $\sqrt{3}$ belongs to $\mathcal{S}$ or to $\mathcal{Z}$. 
We remark that if $\sqrt{3} \in \mathcal{S}$ then writing $\sqrt{3}$ in its base 3 expansion $\sqrt{3}=1+$ $\sum_{j=1}^{\infty} b_{j} 3^{-j}$, where $b_{1}, b_{2}, \ldots \in\{0,1,2\}$, and taking $\xi=1$ we would derive that

$$
\left\{\sqrt{3} \cdot 3^{j}\right\}=b_{j+1} 3^{-1}+b_{j+2} 3^{-2}+\cdots \geq 1 / 2
$$

for infinitely many $j \in \mathbb{N}$. Hence $b_{m}=2$ for infinitely many $m \in \mathbb{N}$. Such results, however, are completely out of reach. See, for instance, [1] for a recent progress on the distribution of digits in the expansions of algebraic irrational numbers in base $b \geq 2$.

The next problem seems to be quite difficult too.

PRoblem 4. Is it true that if $\alpha \in \mathcal{S}$ then for each nonzero real number $\xi$ the sequence $\left[\xi \alpha^{n}\right], n=1,2, \ldots$, contains infinitely many even numbers?

By the definition of $\mathcal{S}$, the sequence $\left[\xi \alpha^{n}\right], n=1,2, \ldots$, contains infinitely many odd numbers. It is easy to see that the answer to Problem 4 is affirmative precisely when there is a nonzero real number $\xi$ such that $\left\{\xi \alpha^{n}\right\} \geq 1 / 2$ for each $n \in \mathbb{N}$, where $\left\{\xi \alpha^{n}\right\}>1 / 2$ for infinitely many $n$ and $\left\{\xi \alpha^{n}\right\}=1 / 2$ for infinitely many $n \in \mathbb{N}$. Taking, for instance, $\xi=1 / 2$ and $\alpha=\sqrt{3}$ we are back to a similar question about the distribution of digits in base 3 expansion again. This time, the number in question is $\sqrt{3} / 2$.

Note that all numbers of $\mathcal{S}$ described in Theorem 2 are algebraic integers and lie in the interval $(1,2)$. We thus conclude this section with the following problem.

Problem 5. Is there an element of $\mathcal{S}$ greater than 2?

3. Proof of Theorem 1. We shall prove (i) and (ii) using the method of nested intervals as in [19]. Suppose first that $\alpha>3$. We claim that there is a $\xi>0$ such that $\left\{\xi \alpha^{n}\right\} \leq \beta:=1 /(\alpha-1)$ for every $n \in \mathbb{N}$. Clearly, $\left\{\xi \alpha^{n}\right\} \leq \beta$ if and only if there is an integer $k_{n}$ such that $k_{n} \alpha^{-n} \leq \xi \leq\left(k_{n}+\beta\right) \alpha^{-n}$. Let $k_{1}$ be an arbitrary integer greater than $\alpha$. Set $I_{1}=\left[k_{1} \alpha^{-1},\left(k_{1}+\beta\right) \alpha^{-1}\right]$. The sequence of intervals $I_{j}=\left[k_{j} \alpha^{-j},\left(k_{j}+\right.\right.$ $\left.\beta) \alpha^{-j}\right]$, where $k_{j} \in \mathbb{N}, j=1,2, \ldots$, is nested if and only if $k_{j} \alpha^{-j} \leq k_{j+1} \alpha^{-j-1}$ and $\left(k_{j}+\beta\right) \alpha^{-j} \geq\left(k_{j+1}+\beta\right) \alpha^{-j-1}$ for each $j \in \mathbb{N}$. This happens precisely when for each $j \in \mathbb{N}$ the interval $\left[\alpha k_{j}, \alpha\left(k_{j}+\beta\right)-\beta\right]$ contains the integer $k_{j+1}$. Since the length of this interval is $\alpha \beta-\beta=1$, such an integer $k_{j+1}$ exists for every $j \in \mathbb{N}$. Hence, setting $\xi:=\cap_{j=1}^{\infty} I_{j}$, we have that $\left\{\xi \alpha^{n}\right\} \leq 1 /(\alpha-1)<1 / 2$ for every $n \in \mathbb{N}$. Therefore, each $\alpha>3$ lies in $\mathcal{Z}$. Trivially, 2, $3 \in \mathcal{Z}$. This proves $(i)$ and also (ii) for $\alpha=2$, where $q=2$.

Next, we will prove (ii) for $\alpha=(3 q-2) / q$, where $q \geq 3$ is an integer. As above, $\left\{\xi(3-2 / q)^{n}\right\} \leq 1 / 2$ if and only if there is an integer $k_{n}$ such that $k_{n}(3-2 / q)^{-n} \leq \xi \leq$ $\left(k_{n}+1 / 2\right)(3-2 / q)^{-n}$. Fix an integer $k_{1}$ greater than 3. Set $I_{1}=\left[k_{1}(3-2 / q)^{-1},\left(k_{1}+\right.\right.$ $\left.1 / 2)(3-2 / q)^{-1}\right]$. The sequence of intervals $I_{j}=\left[k_{j}(3-2 / q)^{-j},\left(k_{j}+1 / 2\right)(3-2 / q)^{-j}\right]$, where $k_{j} \in \mathbb{N}, j=1,2, \ldots$, is nested if and only if

$$
(3-2 / q) k_{j} \leq k_{j+1} \leq(3-2 / q)\left(k_{j}+1 / 2\right)-1 / 2=(3-2 / q) k_{j}+1-1 / q .
$$

It is easy to see that the interval $\left[(3 q-2) k_{j},(3 q-2) k_{j}+q-1\right]$ contains an integer divisible by $q$, say $q u$. So we can take $k_{j+1}:=u$. Hence, setting $\xi:=\cap_{j=1}^{\infty} I_{j}$, we derive that $\left\{\xi(3-2 / q)^{n}\right\} \leq 1 / 2$ for each $n \in \mathbb{N}$. However, since $3-2 / q, q \geq 3$, is not an integer, there are only finitely many $n \in \mathbb{N}$ (or no such $n$ at all) for which $\left\{\xi(3-2 / q)^{n}\right\}=1 / 2$. (See, for instance, Lemma 4 in [11].) If $n_{0}$ is the largest among those $n$ we can replace 
$\xi$ by $\xi(3-2 / q)^{n_{0}}$. With this new $\xi$, the inequality $\left\{\xi(3-2 / q)^{n}\right\}<1 / 2$ holds for every $n \in \mathbb{N}$. This completes the proof of $(i i)$.

For a strong Pisot number $\alpha$, we have $\left\{\alpha^{n}\right\} \rightarrow 1$ as $n \rightarrow \infty$ (see [6]). Indeed, since $S_{n}:=\alpha^{n}+\alpha_{2}^{n}+\cdots+\alpha_{d}^{n} \in \mathbb{Z}$, where $\alpha_{1}=\alpha, \alpha_{2}, \ldots, \alpha_{d}$ are the conjugates of $\alpha$ labelled so that $\alpha>1>\alpha_{2}>\left|\alpha_{j}\right|$ for $j>2$, we deduce that $S_{n}-\alpha^{n}$ is positive for each $n$ sufficiently large. Clearly, $S_{n}-\alpha^{n} \rightarrow 0$ as $n \rightarrow \infty$. It follows that $\left\{\alpha^{n}\right\}=$ $1-\alpha_{2}^{n}-\cdots-\alpha_{d}^{n}$ for each sufficiently large integer $n$. Hence $\left\{\alpha^{n}\right\} \rightarrow 1$ as $n \rightarrow \infty$. In particular, by taking $\xi=-\alpha^{n_{0}}$ with $n_{0}$ sufficiently large, we obtain that $\left\{-\alpha^{n_{0}} \alpha^{n}\right\}<1 / 2$ for each $n \in \mathbb{N}$. This proves (iii).

The proof of (iv) for Pisot and Salem numbers follows [10] and [22], respectively. To be precise, it was shown in [10] that if the minimal polynomial of a Pisot number $\alpha$ satisfies $P_{\alpha}(1) \leq-2$ then, setting $\xi=1 /\left(P_{\alpha}^{\prime}(\alpha)(\alpha-1)\right)$, we have $\lim _{n \rightarrow \infty}\left\{\xi \alpha^{n}\right\}=$ $1 /\left|P_{\alpha}(1)\right|$. Similarly, Zaimi [22] showed that if the minimal polynomial of a Salem number $\alpha$ satisfies $P_{\alpha}(1) \leq-2$ then, for any $\varepsilon>0$, there is a nonzero $\xi=\xi(\alpha, \varepsilon) \in \mathbb{Q}(\alpha)$ such that $1 /\left|P_{\alpha}(1)\right|-\varepsilon<\left\{\xi \alpha^{n}\right\}<1 /\left|P_{\alpha}(1)\right|+\varepsilon$ for each $n \in \mathbb{N}$ large enough. So in both (Pisot and Salem) cases one can find a positive integer $n_{0}$ such that, by taking $\xi \alpha^{n_{0}} \in \mathbb{Q}(\alpha)$ instead of $\xi$, we obtain that $1 /\left|P_{\alpha}(1)\right|-\varepsilon<\left\{\xi \alpha^{n}\right\}<1 /\left|P_{\alpha}(1)\right|+\varepsilon$ for each $n \in \mathbb{N}$. Clearly, this implies the inequality $\left\{\xi \alpha^{n}\right\}<1 / 2$ for each $n \in \mathbb{N}$ under the stronger condition $P_{\alpha}(1) \leq-3$ if $\varepsilon<1 / 6$. This proves (iv). The proof of Theorem 1 is completed.

Since in [10] and in [22] the statements concerning the fractional parts $\left\{\xi \alpha^{n}\right\}$ mentioned in the proof of (iv) are not given explicitly, let us summarize them here as follows.

TheORem 6. Suppose that $\alpha$ is a Pisot number or a Salem number with minimal polynomial $P_{\alpha}(x) \in \mathbb{Z}[x]$. If $P_{\alpha}(1) \leq-2$ then for any $\varepsilon>0$ there is a real number $\xi \in$ $\mathbb{Q}(\alpha)$ (which depends on $\varepsilon$ in the case $\alpha$ is a Salem number) such that

$$
1 /\left|P_{\alpha}(1)\right|-\varepsilon<\left\{\xi \alpha^{n}\right\}<1 /\left|P_{\alpha}(1)\right|+\varepsilon
$$

for any $n \in \mathbb{N}$.

We remark that the fractional parts $\left\{\xi \alpha^{n}\right\}, n \in \mathbb{N}$, can be quite small for some Salem numbers that are not too large. Take, for instance, the Salem number $\alpha=1.6733248 \ldots$ given in [14] whose minimal polynomial is

$$
P_{\alpha}(x)=x^{14}-x^{12}-x^{11}-x^{10}-x^{9}-2 x^{8}-3 x^{7}-2 x^{6}-x^{5}-x^{4}-x^{3}-x^{2}+1,
$$

so that $P_{\alpha}(1)=-13$. Then, by Theorem 6 , for any $\varepsilon>0$, there exists a real number $\xi=\xi(\alpha, \varepsilon) \in \mathbb{Q}(\alpha)$ such that $1 / 13-\varepsilon<\left\{\xi \alpha^{n}\right\}<1 / 13+\varepsilon$ for each $n \in \mathbb{N}$. This not only implies that $\alpha \in \mathcal{Z}$ but also that every integral part $\left[\zeta \alpha^{n}\right]$, where $n \in \mathbb{N}$ and $\zeta=12 \xi$, is divisible by 12 .

4. Proof of Theorem 2. In all three cases it suffices to show that, for any $\xi \neq 0$, the integral parts $x_{n}:=\left[\xi \alpha^{n}\right], n=1,2, \ldots$, cannot all be even. Suppose they are, i.e. $\alpha \in \mathcal{Z}$. Setting $y_{n}:=\left\{\xi \alpha^{n}\right\}$ for $n \in \mathbb{N}$, we have $x_{n+q}-2 x_{n}=2 y_{n}-y_{n+q}$ (case $(i)$ ) or $x_{n+d}-$ $x_{n+r}-x_{n}=y_{n}+y_{n+r}-y_{n+d}$ (case (ii)) or $x_{n+d}-x_{n+m}-x_{n+r}+x_{n}=-y_{n}+y_{n+r}+$ $y_{n+m}-y_{n+d}$ (case (iii)). A fractional part is a non-negative number smaller than 1 . So the right-hand sides of all three equalities belong to the interval $(-2,2)$. But all lefthand sides are even integers. Hence, for every $n \in \mathbb{N}$, we have $x_{n+q}-2 x_{n}=2 y_{n}-y_{n+q}=0$ 
(case $(i)), x_{n+d}-x_{n+r}-x_{n}=y_{n}+y_{n+r}-y_{n+d}=0$ (case (ii)), $x_{n+d}-x_{n+m}-x_{n+r}+$ $x_{n}=-y_{n}+y_{n+r}+y_{n+m}-y_{n+d}$ (case $\left.(i i i)\right)$.

In case $(i)$ we deduce that $y_{n+q m}=2^{m} y_{n}$ for any $m \in \mathbb{N}$. Taking $m$ arbitrarily large we obtain that $y_{n}=\left\{\xi 2^{n / q}\right\}=0$ for every $n \in \mathbb{N}$. Next, by considering, firstly, the subsequence $n=q k, k=1,2, \ldots$, and, secondly, the subsequence $n=q k+1$, $k=1,2, \ldots$, we derive that $\xi 2^{n / q}$ is an integer for every $n \in \mathbb{N}$ if and only if $\xi=0$, a contradiction. Hence $2^{1 / q} \in \mathcal{S}$ for each integer $q \geq 2$. This proves $(i)$.

In case (iii) the sequence $s_{n}:=-y_{n}+y_{n+r}+y_{n+m}-y_{n+d}=0$ is periodic. So, by Lemma 3 of [8], $\alpha>1$ must be a Pisot number or a Salem number. It cannot be a Pisot number by the condition of (iii). Hence $\alpha$ is a Salem number. But from $\alpha^{d}-$ $\alpha^{m}-\alpha^{r}+1=0$ on replacing $\alpha \rightarrow \alpha^{-1}$ (Salem numbers are reciprocal) we obtain that $\alpha^{d}-\alpha^{d-r}-\alpha^{d-m}+1=0$. Observe that if $m+r=d$ then $\alpha^{d}-\alpha^{m}-\alpha^{r}+1=$ $\left(\alpha^{m}-1\right)\left(\alpha^{r}-1\right)=0$, a contradiction with $\alpha>1$. If $m+r \neq d$ then

$$
\begin{aligned}
\alpha^{d}-\alpha^{m}-\alpha^{r}+1-\left(\alpha^{d}-\alpha^{d-r}-\alpha^{d-m}+1\right) & =\alpha^{d-m}-\alpha^{r}+\alpha^{d-r}-\alpha^{m} \\
& =\left(\alpha^{r}+\alpha^{m}\right)\left(\alpha^{d-r-m}-1\right)=0,
\end{aligned}
$$

a contradiction again. This proves (iii). (Note that we proved the following statement: each irreducible reciprocal factor of $x^{d}-x^{m}-x^{r}+1$ is cyclotomic. See [15] for more about irreducible factors of such quadrinomials.)

In case (ii) the sequence $s_{n}:=-y_{n}+y_{n+r}-y_{n+d}=0$ is periodic. As above, Lemma 3 of [8] implies that $\alpha$ is a Pisot number or a Salem number and $\xi \in \mathbb{Q}(\alpha)$. Since $\alpha>1$ is a root of an irreducible nonreciprocal polynomial $x^{d}-x^{r}-1$, it can only be a Pisot number. (Indeed it can: for instance, if $r=1$ and $d=2$ or $d=3$.) Note that it is not a strong Pisot number, because the polynomial $x^{d}-x^{r}-1$ has no roots in the interval $[0,1]$. Suppose that the conjugates of $\alpha=\alpha_{1}>1$ over $\mathbb{Q}$ are $\alpha_{2}, \ldots, \alpha_{d}$, where $\left|\alpha_{1}\right|>1>\left|\alpha_{2}\right| \geq\left|\alpha_{3}\right| \geq \cdots \geq\left|\alpha_{d}\right|$. Since $x_{n+d}-x_{n+r}-x_{n}=0$ for every $n \in \mathbb{N}$, we have that $x_{n}=\xi_{1} \alpha_{1}^{n}+\cdots+\xi_{d} \alpha_{d}^{n}$. Moreover (see [5] or the proof of Theorem 3 in $[8]), \xi_{j} \in \mathbb{Q}\left(\alpha_{j}\right), j=1, \ldots, d$, and the numbers $\xi_{1}, \ldots, \xi_{d}$ are conjugate over $\mathbb{Q}$. Similarly, from the linear recurrence $y_{n+d}-y_{n+r}-y_{n}=0, n=1,2, \ldots$, we obtain that there exist certain complex numbers $\eta_{1}, \ldots, \eta_{d}$ such that $y_{n}=\eta_{1} \alpha_{1}^{n}+\cdots+\eta_{d} \alpha_{d}^{n}$ for each $n \in \mathbb{N}$. But $x_{n}+y_{n}=\xi \alpha_{1}^{n}$, so that $\eta_{1}=\xi-\xi_{1}, \eta_{2}=-\xi_{2}, \ldots, \eta_{d}=-\xi_{d}$. If $\eta_{1} \neq 0$ then $\left|y_{n}\right| \rightarrow \infty$ as $n \rightarrow \infty$, a contradiction. It follows that $\eta_{1}$ must be equal to zero, so $\xi_{1}=\xi$. Summarizing, we have that $y_{n}=-\xi_{2} \alpha_{2}^{n}-\xi_{3} \alpha_{3}^{n}-\cdots-\xi_{d} \alpha_{d}^{n}$, where $\xi_{2} \in \mathbb{Q}\left(\alpha_{2}\right), \ldots, \xi_{d} \in \mathbb{Q}\left(\alpha_{d}\right)$ are conjugate over $\mathbb{Q}$ and $\xi_{2} \neq 0$.

In order to get a contradiction it suffices to show that the sums $y_{n}=-\xi_{2} \alpha_{2}^{n}-$ $\xi_{3} \alpha_{3}^{n}-\cdots-\xi_{d} \alpha_{d}^{n}$ are negative for infinitely many $n \in \mathbb{N}$. Indeed, since every Pisot number $\alpha=\alpha_{1}$ has at most two conjugates of largest modulus in the unit disc (see [18]) which is $\left|\alpha_{2}\right|$, but $\alpha$ is not a strong Pisot number, i.e. $\alpha_{2} \notin(0,1)$ there are only two possibilities. Either $\alpha_{2}$ is a real negative number in $(-1,0)$ and $\left|\alpha_{2}\right|>\left|\alpha_{j}\right|$ for $j>2$ or $\alpha_{2}$ and $\alpha_{3}$ are complex conjugate numbers, i.e. $\alpha_{3}=\overline{\alpha_{2}}$ and $\left|\alpha_{2}\right|>\left|\alpha_{j}\right|$ for $j>3$. In both cases, since $-\xi_{2} \alpha_{2}^{n}-\xi_{3} \alpha_{3}^{n}=-2 \Re\left(\xi_{2} \alpha_{2}^{n}\right)$, the sign of $y_{n}$ is the same as that of $-\Re\left(\xi_{2} \alpha_{2}^{n}\right)$ for each $n$ sufficiently large. Of course, if $\alpha_{2} \in(-1,0)$ then $-\xi_{2} \alpha_{2}^{n}$ is positive for infinitely many $n \in \mathbb{N}$ and negative for infinitely many $n \in \mathbb{N}$. Assume that $\alpha_{2}$ is complex. Let us write $\alpha_{2}=\varrho e^{i \theta}$ and $\xi_{2}=\varrho^{\prime} e^{i \vartheta}$. Then $\Re\left(\xi_{2} \alpha_{2}^{n}\right)=\varrho \varrho^{\prime} \cos (n \theta+\vartheta)$. Since $\theta / \pi$ is irrational (see [17] or derive a contradiction from $\alpha_{2}^{m}=\alpha_{3}^{m}$, where $m \in \mathbb{N}$, by mapping $\alpha_{2}$ to $\alpha$ ), Kronecker's theorem [5] yields that the fractional parts $\{n \theta / 2 \pi+\vartheta / 2 \pi\}, n=1,2, \ldots$, are dense in $[0,1)$. It follows that $\cos (n \theta+\vartheta)$ is positive for infinitely many $n \in \mathbb{N}$ and negative for infinitely many $n \in \mathbb{N}$. This completes the proof of Theorem 2 . 
ACKNOWLEDGEMENTS. This research was supported in part by the Lithuanian State Studies and Science Foundation and by INTAS grant no. 03-51-5070.

\section{REFERENCES}

1. B. Adamczewski and Y. Bugeaud, On the complexity of algebraic numbers I. Expansions in integer bases, Annals of Math., to appear.

2. D. W. Boyd, Linear recurrence relations for some generalized Pisot sequences, in Advances in Number Theory, Proceedings of the 1991 CNTA conference (eds. F. Q. Gouvea and N. Yui) (Oxford University Press, 1993), 333-340.

3. Y. Bugeaud, Linear mod one transformations and the distribution of fractional parts $\left\{\xi(p / q)^{n}\right\}$, Acta Arith. 114 (2004), 301-311.

4. Y. Bugeaud and A. Dubickas, Fractional parts of powers and Sturmian words, $C . R$. Acad. Sci. Paris, Sér. I Math. 341 (2005), 69-74.

5. J. W. S. Cassels, An introduction to diophantine approximation (Cambridge University Press, 1957). $141-144$.

6. A. Dubickas, A note on powers of Pisot numbers, Publ. Math. Debrecen 56 (2000),

7. A. Dubickas, Integer parts of powers of Pisot and Salem numbers, Archiv der Math. 79 (2002), 252-257.

8. A. Dubickas, Arithmetical properties of powers of algebraic numbers, Bull. London Math. Soc. 38 (2006), 70-80.

9. A. Dubickas, On the distance from a rational power to the nearest integer, J. Number Theory 117 (2006), 222-239.

10. A. Dubickas, On the limit points of the fractional parts of powers of Pisot numbers, Archivum Math., to appear.

11. A. Dubickas, On the powers of $3 / 2$ and other rational numbers, submitted.

12. L. Flatto, J. C. Lagarias and A. D. Pollington, On the range of fractional parts $\left\{\xi(p / q)^{n}\right\}$, Acta Arith. 70 (1995), 125-147.

13. J. F. Koksma, Ein mengen-theoretischer Satz über Gleichverteilung modulo eins, Compositio Math. 2 (1935), 250-258.

14. P. Lakatos, A new construction of Salem polynomials, C. R. Math. Rep. Acad. Sci. Canada 25 (2003), 47-54.

15. W. Ljunggren, On the irreducibility of certain trinomials and quadrinomials, Math. Scand. 8 (1960), 65-70.

16. K. Mahler, An unsolved problem on the powers of $3 / 2$, J. Austral. Math. Soc. Ser. A 8 (1968), 313-321.

17. M. Mignotte, Sur les conjugées des nombres de Pisot, C. R. Acad. Sci. Paris, Sér. I Math. 298 (1984), 21.

18. C. J. Smyth, The conjugates of algebraic integers, Amer. Math. Monthly 82 (1975), 86. $1-4$.

19. R. Tijdeman, Note on Mahler's 3/2-problem, K. Norske Vidensk. Selsk. Skr. 16 (1972),

20. T. Vijayaraghavan, On the fractional parts of the powers of a number. I, J. London Math. Soc. (1) 15 (1940), 159-160. 313-352.

21. H. Weyl, Über die Gleichverteilung von Zahlen modulo Eins, Math. Ann. 77 (1916),

22. T. Zaimi, An arithmetical property of Salem numbers, J. Number Theory, to appear.

23. T. Zaimi, On integer and fractional parts powers of Salem numbers, Archiv Math. (Basel), to appear. 